\title{
Heterologous Expression of the Cysteine Proteinase Gene VaCP17from the Cold-adapted Grapevine Vitis Amurensis Increases Cold Tolerance in Arabidopsis
}

\section{Xin Shu}

Northwest Agriculture and Forestry University

\section{Bao Gu}

Northwest Agriculture and Forestry University

Hongjuan Zhang

Northwest Agriculture and Forestry University

\section{Yunyun Tang}

Northwest Agriculture and Forestry University

Jianxia Zhang ( $\square$ zhangjx666@126.com )

Northwest Agriculture and Forestry University

\section{Research Article}

Keywords: Vitis amurensis, VaCP17, transgenic Arabidopsis, cold resistance, interacting proteins

Posted Date: November 2nd, 2021

DOI: https://doi.org/10.21203/rs.3.rs-973595/v1

License: (c) (1) This work is licensed under a Creative Commons Attribution 4.0 International License. Read Full License

Version of Record: A version of this preprint was published at Plant Cell, Tissue and Organ Culture (PCTOC) on February 21st, 2022. See the published version at https://doi.org/10.1007/s11240-02202256-x. 


\section{Abstract}

Cysteine proteinases (thiol) carry out diverse and critical functions in plants through their ability to hydrolyze peptide bonds in target proteins. Here, we cloned a cysteine proteinase gene designated VaCP17 from a highly cold-resistant wild Vitis amurensis accession 'Shuangyou', and then its potential function in cold resistance was investigated. The results showed that the CDS of VaCP17is $1404 \mathrm{bp}$, encoding 467 amino acids, the VaCP17 protein localized to the cell membrane. Expression of CP17 was highly distinctive among different structures of 'Shuangyou' and the cold-sensitive Vitis vinifera cultivar 'Red Globe', with the highest expression in the stem of 'Shuangyou' and the flower of 'Red Globe'. Arabidopsis plants constitutively expressing a VaCP17-GREEN FLUORESCENT PROTEIN fusion (35S:: VaCP17-GFP) showed increased survival after transient exposure to freezing $\left(-6^{\circ} \mathrm{C}\right)$, and showed lower electrolyte leakage and MDA content, higher soluble sugar content and SOD, POD and CAT activities, as compared with non-transgenic Arabidopsis controls. The expression of nine cold-resistance related genes (CBF1, CBF2, CBF3, RD29A, COR15A, KIN1, NCED3, AOC1 and JAZ10) in 35S::VaCP17-GFP plants was increased under cold treatment at $4{ }^{\circ} \mathrm{C}$, relative to control plants. Using a yeast two-hybrid system, we identified VaNAC72, VaCAM7 and VaDi19 as potential interactors of VaCP17, and their interactions were demonstrated by a bimolecular fluorescence complementation assay. In conclusion, we revealed that $\mathrm{VaCP} 17$ can enhance cold resistance by influencing physiology and biochemistry and the expression of cold resistance related genes under cold stress.

\section{Introduction}

Cold is one of the most important abiotic stresses limiting plant geographical distribution and crop production (Ye et al. 2017). The damage caused by low temperature to plants can be classified as cold injury (low temperatures above $0^{\circ} \mathrm{C}$ ) or freezing injury (temperatures below $0^{\circ} \mathrm{C}$ ). Cold stress can destablize the plant cell membrane, increase the accumulation of reactive oxygen species and disrupt protein structure, all of which negatively impact photosynthesis, metabolism, and growth and development (Chinnusamy et al. 2007; Liu et al. 2018; Yin et al. 2021).

Plants have evolved complex regulatory networks that mediate responses to cold stress to increase survival. These include the rapid deployment of the CBF/DREB (C-repeat binding/dehydration element binding) family of transcription factors, which recognize the C-repeat and DRE cis elements in downstream COLD RESPONSIVE (COR) genes to modulate their expression (Shi et al. 2012). The expression of COR genes, such as COR15A, RD29A, KIN1 and GOLS3, can improve plant tolerance to low temperature (Knight et al. 2004; Thomashow 2010; Park et al. 2015; Guo et al. 2019). In addition, cold stress can induce the expression of abscisic acid (ABA) and jasmonic acid (JA) biosynthetic genes, resulting in the accumulation of $A B A$ and $J A$, which also regulate genes required for cold response and survival (Knight et al. 2004; Hu et al. 2013; Feng et al. 2016). Allene oxide cyclase (AOC) is one of the key enzymes in jasmonic acid (JA) biosynthesis, which can affect the stereoisomerization and biological activity of jasmonic acid molecules, and plays an important role in plant stress resistance (Sun et al. 2020). Jasmonic acid induces the degradation of JAZ protein and activates the expression of cold 
resistance-related genes, thus improving plant cold resistance (An et al. 2021). Abiotic stresses such as cold, salt and drought usually increase the content of $A B A$, which in turn induces stomatal closure and reduces transpiration rate; $\mathrm{ABA}$ can also regulate the expression of stress-related genes (Mulholland et al. 2003; Jiang et al. 2014; Li et al. 2019). The rate-limiting enzyme of ABA biosynthesis is 9-cisepoxycarotenoid dioxygenases (NCEDs). Together, these mechanisms act to enhance the stability of cellular membranes by promoting accumulation of osmolytes such as proline, soluble sugar and soluble protein (Ashraf and Foolad 2007).

Cysteine proteinases, also known as thiol or sulfhydryl proteinases, play various roles in plant growth and development, including senescence and programmed cell death (PCD) (Xu et al. 2003; Paireder et al. 2016; Burke et al. 2020). Senescence can be conditioned by several factors, including water deficit, phytohormones, and pathogen infection (Yang et al. 2003; Munné-Bosch and Alegre 2004; Mercedes et al. 2016). Likewise, PCD can be triggered by a variety of biotic and abiotic stresses (Paireder et al. 2016; Burke et al. 2020). Both abiotic and biotic stress can lead to damage of intracellular proteins, and proteolysis of damaged proteins by cysteine proteinases can minize the potentially negative effects of aberrant protein activity (García-Lorenzo et al. 2006; López-Barón et al. 2017). Abiotic stresses will aggravate the production of reactive oxygen species (ROS), and the excessive accumulation of ROS will lead to oxidative stress, leading to various metabolic disorders and affecting plant growth and development (Bose et al. 2014). Cysteine proteinases are up-regulated under oxidative stress and play an important role in maintaining cell metabolism (Usui et al. 2007). Cysteine proteinases can also regulate the level of reactive oxygen species in cells, thus reducing oxidative damage to membranes and proteins (Hoorn and Renier 2008).

Several studies have revealed that cysteine proteinase genes can be induced by abiotic stress (Jones and Mullet. 1995; Zang et al. 2010; Zheng et al. 2018). For example, in Arabidopsis thaliana (Arabidopsis), Pisum sativum, Nicotiana tabacum and Ipomoea batatas, cysteine proteinase genes are upregulated in response to drought, salt, cold or exogenous hormones such as gibberellin, jasmonic acid and abscisic acid (Koizumi et al. 1993; Jones and Mullet 1995; Beyene 2006; Chen et al. 2010). Zhang et al. exposed wheat seedlings to low temperature, high temperature, salt and drought stresses, and found that wheat TaCP3 was induced by all these conditions, especially drought (Zhang et al. 2019).

Ectopic expression of the cysteine protease $S p C P 3$ from sweet potato in transgenic Arabidopsis enhanced the sensitivity of the plants to drought stress (Chen et al. 2013). Heterologous expression of Salix matsudana SmCP in Arabidopsis improved salt tolerance, and this was associated with increased stability of cell membrane and activity of the antioxidant enzyme system (Zheng et al. 2018). Therefore, plant cysteine proteases play a key role in the defense to abiotic stress.

Vitis amurensis (Amur grape) is native to present-day northeastern China and far-eastern Russia, and is adapted to extremely low winter temperatures. Currently there is wide interest in exploiting genes/alleles conferring cold tolerance in $V$. amurensis for cultivar improvement of domestic grapes, including the commerically valuable Vitis vinifera. In this study, we identified a cysteine proteinase gene from $V$. 
amurensis that is induced in response to cold. We generated transgenic Arabidopsis expressing VaCP17, and analyzed phenotypic, physiological and biochemical indices related to cold stress response, and evaluated expression of cold-resistance related genes. We further identified proteins that could interact with VaCP17 by yeast two-hybrid technology. Together, the results of these experiments lay the foundation for further studies of the molecular mechanisms of VaCP17 in cold resistance of Vitis amurensis.

\section{Materials And Methods}

\subsection{Plant materials and treatments}

Vitis amurensis acc. 'Shuangyou' and Vitis vinifera cv. 'Red Globe' were maintained in the grapevine germplasm repository at Northwest A\&F University, Yangling, Shaanxi, China. Seeds of Arabidopsis thaliana and Nicotiana benthamiana were obtained from the State Key Laboratory of Crop Stress Biology in Arid Areas, Northwest A\&F University.

To assess freezing tolerance of Arabidopsis, plants were maintained in a controlled environment chamber at $10^{\circ} \mathrm{C}$ for $24 \mathrm{~h}$, and then the temperature was changed to $-6^{\circ} \mathrm{C}$. To assess physiological and biochemical indices, another set of plants were acclimated at $10^{\circ} \mathrm{C}$ for $24 \mathrm{~h}$ and then subjected to $4^{\circ} \mathrm{C}$ for $48 \mathrm{~h}$. Samples were taken respectively at $0,3,6,12,24$ and $48 \mathrm{~h}$. Three biological replicates were included at each time point. Samples were immediately stored at $-80^{\circ} \mathrm{C}$.

\subsection{Cloning and sequence analysis of VaCP17gene}

The VaCP17 coding sequence (CDS) was cloned by PCR from cDNA derived from leaves of Vitis amurensis acc. 'Shuangyou', using oligonucleotide primers VaCP17-F and VaCP17-R as designed by Primer Premier 5.0 software (Supplementary table 1). The PCR reaction contained PrimeSTAR® (Takara) Buffer, $200 \mu \mathrm{M}$ dNTPs, $0.4 \mu \mathrm{M}$ primer VaCP17-F, 0.4 $\mu \mathrm{M}$ primer VaCP17-R, $2 \mu \mathrm{L}$ cDNA, and 1.25 units PrimeSTAR ${ }^{\circ} \mathrm{HS}$, in a total volume of $50 \mu \mathrm{L}$. The reaction profile was 32 cycles of $98^{\circ} \mathrm{C}$ for $10 \mathrm{~s} ; 57^{\circ} \mathrm{C}$ for $15 \mathrm{~s}$, and $72^{\circ} \mathrm{C}$ for $1 \mathrm{~min} 30 \mathrm{~s}$, with a final incubation at $72^{\circ} \mathrm{C}$ for $10 \mathrm{~min}$. Amplified DNA was purified and cloned using the pMD19-T vector to generate PMD19-T-VaCP17. The VaCP17CDS in pMD19-T-VaCP17 was subjected to Sanger sequencing on both strands to ensure that no mutations had resulted from the PCR amplification.

A draft genome annotation of Vitis amurensis was used to determine the chromosomal location of VaCP17 (Wang et al. 2021). Homologous sequences were identified from the NCBI nr (non-redundant protein) database using BLASTP and the predicted VaCP17 amino acid sequence as a query. Multiple sequence alignments and phylogenetic reconstructions utilized DNAMAN and MEGA7.0, respectively.

\subsection{VaCP17 subcellular localization assay}

The cloned VaCP17CDS was inserted into the pCAMBIA2300 vector downstream of the 35 S promoter to generate the 35S:: VaCP17-GFP expression vector. 35S::VaCP17-GFP and pCAMBIA2300 plasmid DNAs 
were introduced into Arabidopsis protoplasts by a polyethylene glycol (PEG)-mediated method (Yu et al., 2013). The protoplasts were cultured at $22^{\circ} \mathrm{C}$ under weak light for about $18 \mathrm{~h}$. The localization of VaCP17-GFP fusion protein was observed by confocal microscopy (LEICA TCS SP8, Leica, Germany).

\subsection{Analysis of $C P 17$ expression in structures of 'Shuangyou' and 'Red Globe'}

Young roots, stems, leaves, flowers and tendrils of 'Shuangyou' and 'Red Globe' were collected from plants growing under natural conditions in the field. RNA was extracted and first-strand cDNA generated using the E.Z.N.A. Plant RNA Kit from Omega Bio-tek and FastQuant RT with gDNAase from TIANGEN, respectively. qRT-PCR analysis was performed using SYBRßPremix Ex TaqTMII (ThermoFisher) and the QS6 real-time quantitative PCR detection system (Bio-Rad). RT-CP17-F and RT-CP17-R were used as primers (Supplementary table 1), and VvActin (XM_002265440) was employed as the internal reference gene.

\subsection{Production and identification of transgenic Arabidopsis expressing VaCP17}

\subsubsection{Production of VaCP17transgenic Arabidopsis}

Agrobacterium tumefaciens strain GV3101 harboring 35S::VaCP17-GFP was used to transform Arabidopsis plants by the floral dip method. $T 1$ seedlings resistant to Kan were identified by germination and growth on $1 / 2 x$ MS medium containing $50 \mathrm{mg} / \mathrm{L}$ Kan. Homozygous lines were identified by progeny testing, and initiated from $\mathrm{T} 2$ plants.

\subsubsection{Identification of VaCP17transgenic Arabidopsis}

Genomic DNA was extracted from rosette leaves of T3 homozygous plants, and the presence of pCAMBIA2300 sequence was determined using PCR and primers specific for pCAMBIA2300 (Supplementary table 1). PCR-positive plants were evaluated for VaCP17 expression using real-time fluorescence quantitative RT-PCR and gene-specific primers as shown in Supplementary table 1.

To detect VaCP17 protein, total proteins were extracted from Arabidopsis leaves using the method described by Wang et al. (2006), separated on a 10\% SDS-PAGE gel and transferred to PVDF film using a semi-dry transfer membrane method. Anti-GFP-tagged mouse monoclonal antibody (TransGen Biotech) was used as the primary antibody and a HRP-labeled goat anti-mouse $\lg \mathrm{G}(\mathrm{H}+\mathrm{L})$ (TransGen Biotech) was used as the secondary antibody.

\subsection{Evaluation of tolerance of 35S:VaCP17 transgenic Arabidopsis to cold stress}

\subsubsection{Phenotypic changes of transgenic plants under cold stress}


Seeds of 35S::VaCP17-GFPlines or non-transgenic control plants were sown in an artificial potting medium, and seedlings/plants were cultivated for four weeks at $22^{\circ} \mathrm{C}$ under $16 \mathrm{~h}$-light/8h-dark photoperiods. Subsequently plants were transferred to $10^{\circ} \mathrm{C}$ for $24 \mathrm{~h}$, and then $-6^{\circ} \mathrm{C}$ for $8 \mathrm{~h}$. After the freezing treatment, the temperature was raised at $3^{\circ} \mathrm{C} / \mathrm{h}$ to $22^{\circ} \mathrm{C}$, and then plants were returned to previous conditions for $10 \mathrm{~d}$ prior to phenotypic observations.

\subsubsection{Physiological and biochemical indices of 35S:: VaCP17-GFP plants under cold stress}

Leaves of 35S:: VaCP17-GFP and non-transgenic control plants lines were removed after cold treatment and used to evaluate electrolyte leakage, contents of MDA, proline and soluble sugar, and activities of SOD, POD, and CAT, as described by Gao (2006).

2.6.3 Analysis of expression of VaCP17 and cold-resistance-related genes in 35S::VaCP17-GFP and control plants under cold stress.

Four-week-old 35S:: VaCP17-GFP and non-transgenic control plants were placed at $10^{\circ} \mathrm{C}$ for $24 \mathrm{~h}$, then at $4^{\circ} \mathrm{C}$ for $48 \mathrm{~h}$, and samples were taken at $0,3,6,12,24$ and $48 \mathrm{~h}$, with three biological replicates at each time point. RNA was extracted from the samples and subjected to reverse transcription into cDNA. AtACTIN (AT3G18780) was used as an internal reference gene. Primers used for quantitiative analysis of expression of $\mathrm{VaCP} 17$ and cold resistance-related genes are shown in Supplementary table 1.

\subsection{Screening and verification of VaCP17-interacting proteins}

\subsubsection{Verification of VaCP17 self-activation}

The VaCP17CDS was cloned by PCR using primers described in Supplementary table 1 and inserted into pGBKT7 (Supplementary table 1). The pGBKT7/VaCP17 plasmid was extracted and transferred into yeast $\mathrm{Y} 2 \mathrm{HGold}$ cells by the LiAc method, and tranformed cultures were spread onto SD/-Trp agar medium. After incubating at $30^{\circ} \mathrm{C}$ for $3 \mathrm{~d}, \mathrm{P} 53 /$ pGBKT7 and Lam/pGBKT7 were co-transformed with pGADT7-T, and cultures were spread on SD/-Trp-Leu medium as positive and negative controls, respectively. Three isolates each for pGBKT7/VaCP17, negative and positive controls were selected and suspended in $150 \mu \mathrm{l}$ sterile water, then spotted onto SD/-Trp medium containing $200 \mathrm{ng} / \mathrm{ml}$ AbA and 20 $\mathrm{mg} / \mathrm{ml} \mathrm{X-a-gal.}$

\subsubsection{Identification of VaCP17-interacting proteins by yeast two-hybrid screening}

A cDNA library was constructed RNA derived from leaves of a Vitis amurensis acc. 'Shuangyou' individual that had been subjected to $0^{\circ} \mathrm{C}$. The library was constructed in the plasmid pGADT7, and the VaCP17 CDS was cloned into the bait vector plasmid pGBKT7/VaCP17. Prey and bait vectors were cotransformed into Y2HGold yeast cells, spread on SD/-Trp/-Leu/-Ade/-His solid medium, and positive 
clones were characterized by DNA sequencing. In order to confirm initially observed protein interactions, selected candidate genes were cloned independently into PGADT7 and co-transformed with the pGBKT7/VaCAP17 bait vector into Y2HGold cells, and then spread onto SD/-Trp/-Leu solid medium. After $3 \mathrm{~d}$ of growth, clones were selected and verified on SD/-Trp/-Leu/-Ade/-His/AbA/X-a-gal solid medium.

\subsubsection{Bimolecular fluorescence complementation assay for VaCP17-interacting proteins}

The CDS sequence of $\mathrm{VaCP} 17$ was incorporated into the PBI221NE vector by homologous recombination to generate $\mathrm{PBI} 221 \mathrm{NE} / \mathrm{VaCP} 17$, and the CDS sequence of interacting proteins were incorporated into $\mathrm{PBI} 221 \mathrm{CE}$ to generate PBI221 CE/X, using oligonucleotide primers as listed in Supplementary table 1. The $\mathrm{PBI} 221 \mathrm{NE} / \mathrm{VaCP} 17$ and PBI221CE/X plasmids were extracted and co-transformed into Arabidopsis protoplasts by a polyethylene glycol (PEG)-mediated method (Yu et al. 2013). The protoplast suspension was then cultured at $22^{\circ} \mathrm{C}$ under weak light for about $18 \mathrm{~h}$. YFP fluorescence was observed with a confocal microscope (LEICATCS SP8, Leica, Germany), using an excitation wavelength of $514 \mathrm{~nm}$ and emission wavelength of $527 \mathrm{~nm}$.

\subsection{Statistical analysis}

Data are presented here as the mean \pm standard deviation (SD) of three biological replicates. All data were analyzed by LSD and Duncan methods of SPSS one-way ANOVA $\left({ }^{\star} P<0.05 ; * \star P<0.01 ; * \star * P<0.001\right)$.

\section{Results And Analysis}

\subsection{Cloning of VaCP17 and phylogenetic analyses}

As a first step to assess a potential role for VaCP17 in cold tolerance, we cloned a 1404-bp, full-length VaCP17 coding sequence (CDS) using RT-PCR and RNA from 'Shuangyou' leaf as template (Fig. 1a). According to the genome of Vitis amurensis, the VaCP17 gene is located on Chromosome 18 (Fig. 1b). The presumed VaCP17 protein contains three conserved domains: Inhibitor_I29, Peptidase_C1A and GRAN (Fig. 1b). Homologous sequences to VaCP17 in Arabidopsis thaliana, Carica papaya, Citrus sinensis, Durio zibethinus, Hevea brasiliensis, Juglans regia, Malus domestica, Nicotiana tabacum, Prunus avium, Populus trichocarpa, Pistacia vera, Ziziphus jujuba were identified in the NCBI nr database, and the amino acid sequences of VaCP17 and CPs with high homology were compared. It was found that the amino acid sequence similarity between VaCP17 and CsCP was the highest, at $80.6 \%$ (Fig. 1c). Results of a phylogenetic analysis including the most closely homologous sequences showed that VaCP17 was most closely related to CPs from Nicotiana tobacum and Carica papaya (Fig. 1d).

\subsection{VaCP17 localises to the cell membrane}

To determine the subcellular localization of VaCP17, the 35S::VaCP17-GFP construction in pCAMBIA2300, or the non-modified pCAMBIA2300 plasmid, were transformed into Arabidopsis 
protoplasts. The results showed that green fluorescence was observed on cell membrane, while in the control green fluorescence was visible in the whole cell. This suggests that VaCP17 localizes to the cell membrane.

\subsection{Developmental expression of CP17 in 'Shuangyou' and 'Red Globe'}

We evaluated the developmental expression of $C P 17$ in five structures (root, stem, leaf, flower, and tendril) of 'Shuangyou' and 'Red Globe' plants growing under field conditions. CP17 expression was detectable in all of the structures in both genotypes. In 'Shuangyou', expression was highest in the stem, while in 'Red Globe' expression was relatively low in the stem and highest in the flower (Fig. 3). This finding suggests that the $C P 17$ gene is regulated distinctly between the two genotypes during development.

\subsection{Production and molecular identification of transgenic plants}

To assess the potential effects of heterologous expression of $\mathrm{VaCP} 17$ in Arabidopsis, we engineered a VaCP17 fusion with the GFP sequence under control of the strong, consistutive 35S promoter and introduced this into Arabidopsis via Agrobacterium-mediated transformation. Ten independent T1 lines were initially analyzed by QRTPCR, using oligonucleotide primers specific for the VaCP17CDS (Fig. 4a, b), and immunoblotting using anti-GFP antibody (Fig. 4c). Western blot analysis revealed an immunoreactive species of the expected VaCP17-GFP molecular mass ( 76 kDa) in lines OE-1, OE-3 and OE-5, and these three lines were further evaluated (Fig. 4c). Based on these analyses, we selected three strongly expressing T1 individuals (OE-1, OE-3 and OE-5) and established homozygous lines for further study.

\subsection{Overexpression of VaCP17 confers cold resistance in Arabidopsis}

We noticed no striking difference in growth or development between 35S: VaCP17-GFP transgenic plants and non-transgenic controls when maintained at $22^{\circ} \mathrm{C}$ (Fig. 5a). However, when plants were subjected to a freezing treatment of $-6^{\circ} \mathrm{C}$ for $8 \mathrm{~h}$, non-transgenic controls showed severe wilting, whereas 35S:: VaCP17-GFP plants showed only mild wilting. After a 10 -d recovery period at $22^{\circ} \mathrm{C}$, almost all control plants died, while 35S::VaCP17-GFP lines quickly resumed normal growth (Fig. 5a). This result suggested that expression of $\mathrm{VaCP} 17$ significantly increased the resistance of Arabidopsis to transient freezing. Electrolyte leakage (EL) and malondialdehyde content (MDA) are two indicators of cell damage caused by freezing. After the freezing treatment, the contents of EL and MDA in both control and 35S:: VaCP17-GFP plants increased, but the increases in control plants were much greater than in the 35S:: VaCP17-GFPlines (Fig. 5b, c). These results indicated that the increased freezing tolerance presumably conferred by $V a C P 17$ was associated with reduced cellular damage. 
Plants under cold stress can increase synthesis of proline and soluble sugar as osmolytes, enhancing their water retention ability and cold resistance (Ashraf and Foolad 2007). As shown in Fig. 5 (d, e), both control and 35S:: VaCP17-GFP plants accumulated proline and soluble sugar during the cold treatment. However, proline and soluble sugars both accumulated to higher levels in the 35S::VaCP17-GFP plants relative to the controls; this difference was most striking, and highly significant, for soluble sugars. In addition, we also evaluated the activities of three antioxidase enzymes that are known to be upregulated in plants under stress-superoxide dismutase (SOD), peroxidase (POD), and catalase (CAT). All three showed increased activity in the cold-treated plants; however, the increase in activities was significantly greater for the 35S:: VaCP17-GFP lines (Fig. 5f-h). These results suggest that the active oxygen scavenging system in the 35S:: VaCP17-GFP plants was more active under cold stress. In summary, the results of these physiological and biochemical experiments demonstrate that VaCP17 could confer increased cold tolerance to Arabidopsis.

\subsection{VaCP17 promotes cold resistance-related genes expression in Arabidopsis}

To further explore the mechanism by which VaCP17 promotes cold tolerance, we analyzed the relative expression of VaCP17 and nine cold-resistance related genes (CBF1, CBF2, CBF3, RD29A, COR15A, KIN1, NCED3, AOC1 and JAZ10) in the 35S:: VaCP17-GFP and control plants at various times after plants were moved to the cold $\left(4^{\circ} \mathrm{C}\right)$. Among them, CBF1, CBF2 and CBF3 are early cold-response genes, while RD29A, COR15A and KIN1 respond later to cold. AOC1 and JAZ10 are related to JA synthesis, and NCED3 is related to ABA synthesis (Jiang et al. 2014; Sun et al. 2020; An et al. 2021). Interestingly, although VaCP17 was expressed under control of the constitutive 35S promoter, expression of VaCP17increased rapidly during the cold treatment, reaching a maximum at $6 \mathrm{~h}$ and then declining to near-initial levels by $24 \mathrm{~h}$ (Fig. 6). All of the nine cold-responsive genes were also induced during the cold treatment in both 35S:: VaCP17-GFP and non-transgenic control plants (Fig. 7), as anticipated. However, each gene was expressed to significantly higher levels in the representative 35S:: VaCP17-GFP line tested, at least one time point. These observations suggests that VaCP17increased the cold resistance of Arabidopsis by promoting cold-responsive gene expression and participating in the JA and ABA pathways.

\subsection{Identification of VaCP17-interacting proteins}

To further our knowledge of potential mechanisms by which VaCP17 may promote cold tolerance in grapevine, we used the yeast two-hybrid approach to identify proteins that interact with VaCP17. Yeast colonies containing VaCP17/pGBKT7 did not grow on the SD/-Trp/AbA/X-a-gal medium and did not develop a blue color (Fig. 8a), indicating that VaCP17 had no independent transcriptional activation activity and could be used for subsequent cDNA library screening. Three candidate genes VaNAC72, VaDi19 and VaCAM7 were obtained after co-transformation of bait vector plasmid VaCP17/pGBKT7 and library plasmid constructed from the leaves of cold-treated 'Shuangyou'. All three candidate genes were inserted into the pGADT7 vector and co-transformed into Y2HGold yeast cells with VaCP17/pGBKT7 for 
verification (Fig. 8b). These results confirmed that VaCP17 could interact with VaNAC72, VaDi19 and VaCAM7 in yeast cells.

\subsection{VaCP17 interacts with VaNAC72, VaDi19 and VaCAM7 in vivo}

We tested whether these three proteins could interact with VaCP17 in vivo using bimolecular fluorescence complementation (BiFC). The CDS sequence of VaCP17 was inserted into the PBI221NE vector to generate PBI221NE/VaCP17. At the same time, the CDS sequences of VaNAC72, VaDi19 and VaCAM7 were inserted into PBI221CE to generate PBI221CE/VaNAC72, PBI221CE/VaDi19 and $\mathrm{PBI} 21 \mathrm{CE} / \mathrm{VaCAM7}$. As shown in figure 8c, YFP signal could be observed upon co-transformation of PBI221NE/VaCP with PBI221CE/VaNAC72, PBI221CE/VaDi19 or PBI221CE/VaCAM7, while no YFP signal was observed after co-trasnforamtion of PBI221NE/VaCP with the non-modified PBI221CE plasmid. These results supported a physical interaction of VaCP17 with VaNAC72, VaDi19 and VaCAM7 in vivo.

\section{Discussion}

Cysteine proteinases play important roles in plant growth and development, senescence and programmed cell death, and it has been reported that they may participate in response to various stresses (Xu et al. 2003; Zang et al. 2010; Zheng et al. 2018). However, to date, there have been no reported studies of cysteine proteinases in grapevines. In this study, we explored the potential position of VaCP17in mechanisms of cold and freezing tolerance of Vitis amurensis. Similar to cysteine proteases identified in other species, the VaCP17 protein contains three conserved domains, Inhibitor_I29, Peptidase_C1A and GRAN, indicating that it belongs to the PapainC1A family (Drenth et al. 1968; Rawlings and Barrett 2010). The phylogenetic tree shown in Fig. 1 indicates that VaCP17 is closely related to AtCP from Arabidopsis. In Arabidopsis, the AtRD19A and AtRD21 genes encode cysteine proteinases included in the papain family, and can be upregulated in response to drought or salt stress (Masahiro et al. 1993). So we speculated that $\mathrm{VaCP} 17$ may be involved in response to cold stress in grape.

Interestingly, we found that VaCP17 expression in 35S::VaCP17-GFP plants increased in response to cold, even though the gene was under control of the constitutive 35 S promoter (Fig. 6). This suggests that VaCP17 expression is promoted through post-transcriptional mechanisms in Arabidopsis. Compared with non-transgenic control plants, the three independent Arabidopsis 35S:: VaCP17-GFPlines showed better survival after a short freezing treatment, with higher proline and soluble sugar content, higher antioxidant activities, and lower MDA and EL (Fig. 5). In this study, the activities of three antioxidant enzymes (SOD, POD and CAT) in three transgenic lines were significantly higher than those in the non-transgenic control plants, indicating that $\mathrm{VaCP} 17$ is able to recruit the antioxidant enzyme system to scavenge ROS. This finding is similar to the previous demonstration that heterologous expression of a cysteine proteinase from Salix matsudana in Arabidopsis was sufficient to enhance the antioxidant enzyme system (Zheng et al. 2018). 
Plants respond to adverse environmental conditions by modulating the expression of many stressresponsive genes. To further explore the molecular mechanism by which VaCP17 promotes cold tolerance, we analyzed the expression of nine cold-resistance related genes under low temperature treatment at $4^{\circ} \mathrm{C}$. In this study, the expression of $C B F$ genes, as well as RD29A, COR15A and KIN1, were induced to higher levels in 35S::VaCP17-GFP transgenic plants relative to non-transgenic control plants. In addition, $J A$ and $A B A$ synthesis genes and signal pathway genes were significantly induced in 35S:: VaCP17-GFP. Therefore, we speculate that VaCP17 may be involved in responding to JA and ABA signaling pathways and respond to cold stress by cooperating with these cold resistance related genes, thereby significantly improving the cold resistance of Arabidopsis.

To further reveal the mechanism by which VaCP17 promotes cold resistance, three proteins (VaNAC72, VaDi19 and VaCAM7) interacting with VaCP17 were identified by $\mathrm{Y} 2 \mathrm{H}$ and BiFC (Fig. 8). NAC is one of the largest transcription factor families in plants (Puranik et al. 2012), and individual NAC proteins are involved in responses to various biotic and abiotic stresses (Takada et al. 2001; Mallory et al. 2004; Liu et al. 2014). For example, transgenic rice overexpressing ONACO22 are more tolerant to drought and salt stress (Hong et al. 2016). The strawberry FaNAC2 gene could be significantly induced by cold, salt and drought stresses, and transgenic tobacco expressing FaNAC2 showed increased resistance to cold and salt stresses (Liang et al. 2020). Di19 proteins are a subset of the Cys2/His2 zinc finger protein family, which plays an important role in regulating multiple stress responses (Milla et al. 2006; Qin et al. 2014). In Arabidopsis, AtDi19-1 and AtDi19-3 could be rapidly induced by drought stress, while AtDi19-2 and AtDi194 could be induced by salt stress (Milla et al. 2006). In addition, GhDi19-1 and GhDi19-2 are involved in plant response to salt stress and ABA signals (Li et al. 2010).

Calcium plays a key role in plant development and abiotic stress response as a second messenger (Dodd et al. 2010; Kudla et al. 2018). Plants complete the signal transduction process by increasing the concentration of intracellular calcium, which activates a complex intracellular defense mechanism (Yang et al. 2003). The cold tolerance of transgenic tomato overexpressing SICML37 was significantly improved. The conformation of SICML37 induced by $\mathrm{Ca}^{2+}$ could interact with SIUMP1; activated SIUMP1 could affect the activity of proteasome, and regulate the degradation of target proteins, which improve plant cold tolerance (Tang et al. 2021). Therefore, it is speculated that the interaction between VaCP17 and NAC72, Di19 and CAM7 may affect the expression of downstream genes, and then jointly regulate the tolerance of plants to cold stress.

In conclusion, the results of this study indicate that $\mathrm{VaCP} 17$ can enhance the tolerance of transgenic Arabidopsis to cold stress. However, more work is needed to clarify the underlying molecular mechanism.

\section{Declarations}

Acknowledgments: This study was supported by Shaanxi Province Key Project-Agriculture of the People's Republic of China [grant number 2017ZDXM-NY-026], and The National Science-Technology Support 
Plan Projects of the Ministry of Science and Technology of the People's Republic of China [grant number 2013BAD02B04-06].

Author Contributions: ZJX designed the experiments and revised the manuscript. SX performed experiments and wrote the manuscript. GB cloned VaCP17. ZHJ participated in RNA extraction from the 35S:: VaCP17-GFPlines. TYY participated in the determination of physiological and biochemical indicators.

Data availability: The data sets supporting the results of this article are included within the article and its additional files.

Conflict of interest: The authors declare that the research was conducted in the absence of any commercial or fnancial relationships that could be construed as a potential confict of interest.

\section{References}

1. An JP, Wang XF, Zhang XW, You CX, Hao YJ (2021) Apple B-box protein BBX37 regulates jasmonic acid mediated cold tolerance through the JAZ-BBX37-ICE1-CBF pathway and undergoes MIEL1mediated ubiquitination and degradation. New Phytol 229:2707-2729

2. Ashraf M, Foolad MR (2007) Roles of glycine betaine and proline in improving plant abiotic stress resistance. Environ Exp Bot 59:206-216

3. Beyene $G$ (2006) Two new cysteine proteinases with specific expression patterns in mature and senescent tobacco (Nicotiana tabacum L.) leaves. J Exp Bot 57:1431-1443

4. Burke R, Schwarze J, Sherwood OL, Jnaid Y, McCabe PF, Kacprzyk J (2020) Stressed to Death: The role of transcription factors in plant programmed cell death induced by abiotic and biotic stimuli. Front Plant Sci 11:1235

5. Chen HJ, Su CT, Lin CH, Huang GJ, Lin YH (2010) Expression of sweet potato cysteine protease $S P C P 2$ altered developmental characteristics and stress responses in transgenic Arabidopsis plants. J Plant Physiol 167:838-847

6. Chen HJ, Tsai YJ, Shen CY, Tsai TN, Huang GJ, Lin YH (2013) Ectopic expression of sweet potato cysteine protease SPCP3 alters phenotypic traits and enhances drought stress sensitivity in transgenic Arabidopsis plants. J Plant Growth Regul 32:108-121

7. Chinnusamy V, Zhu J, Zhu JK (2007) Cold stress regulation of gene expression in plants. Trends Plant Sci 12:444-451

8. Dodd A, Kudla J, Sanders D (2010) The language of calcium signaling. Annu Rev Plant Biol 61:593620

9. Drenth J, Jansonius JN, Koekoek R, Swen HM, Wolthers BG (1968) Structure of papain. Nature 218:929-932

10. Feng W, Guo ZX, Li HZ, Wang, MM, Onac E, Zhou J, Xia XJ, Shi K, Yu JQ, Zhou YH (2016) Phytochrome A and B Function Antagonistically to Regulate Cold Tolerance via Abscisic Acid- 
Dependent Jasmonate Signaling. Plant Physiol 170:459-471

11. Gao JF (2006) Plant physiology experiment guidance. Higher Education Press, Beijing, China (in Chinese)

12. García-Lorenzo M, Sjödin A, Jansson S, Funk C (2006) Protease gene families in Populus and Arabidopsis. BMC Plant Biol 6:30

13. Guo J, Ren Y, Tang ZH, Shi WP, Zhou MX (2019) Characterization and expression profiling of the ICECBF-COR genes in wheat. Peer J 7:e8190

14. Hong YB, Zhang HJ, Huang L, Li DY, Song FM (2016) Overexpression of a stress-responsive NAC transcription factor gene ONAC022 improves drought and salt tolerance in rice. Front Plant Sci 7:119

15. Hoorn VD, Renier AL (2008) Plant Proteases: From phenotypes to molecular mechanisms. Annu Rev Plant Biol 59:191-223

16. Hu Y, Jiang L, Wang F, Yu D (2013) Jasmonate regulates the inducer of cbf expression-C-repeat binding factor/DRE binding factor1 cascade and freezing tolerance in Arabidopsis . Plant Cell 25:2907-2924

17. Jiang Y, Wu K, Lin F, Qu YN, Liu XX, Zhang Q (2014) Phosphatidic acid integrates calcium signaling and microtubule dynamics into regulating ABA-induced stomatal closure in Arabidopsis. Planta 239:565-575

18. Jones JT, Mullet JE (1995) A salt- and dehydration-inducible pea gene, Cyp 15a, encodes a cell-wall protein with sequence similarity to cysteine proteases. Plant Mol Biol 28:1055-1065

19. Knight H, Zarka DG, Okamoto H, Thomashow MF, Knight MR (2004) Abscisic acid induces CBF gene transcription and subsequent induction of cold-regulated genes via the CRT promoter element. Plant Physiol 135:1710-1717

20. Koizumi M, Yamaguchi-Shinozaki K, Shinozaki K (1993) Structure and expression of two genes that encode distinct drought-inducible cysteine proteinases in Arabidopsis thaliana. Gene 129:175-82

21. Kudla J, Becker D, Grill E, Hedrich R, Hippler M, Kummer U, Parniske M, Romeis T, Schumacher K (2018) Advances and current challenges in calcium signaling. New Phytol 218:414-431

22. Li G, Tai FJ, Zheng Y, Luo J, Gong SY, Zhang ZT, Li XB (2010) Two cotton Cys2/His2-type zinc-finger proteins, GhDi19-1 and GhDi19-2, are involved in plant response to salt/drought stress and abscisic acid signaling. Plant Mol Biol 74:437-452

23. Li T, Sun JK, Li CR, Lu ZH, Xia JB (2019) Cloning and expression analysis of the FvNCED3 gene and its promoter from ash (Fraxinus velutina). J Forestry Res 30:471-482

24. Li JB, Zheng GS, Li SF, Tang ZM, Sun JS, Xin XB (2020) Characterization of Rosa chinensis CBF genes and the function of RcCBF6 in Cold Tolerance. J Plant Biol 63:267-278

25. Liang JH, Zheng J, Wu Z, Wang HQ (2020) Strawberry FaNAC2 enhances tolerance to abiotic stress by regulating proline metabolism. Plants-basel 9:1417 
26. Liu J, Peng T, Dai W (2014) Critical cis-acting elements and interacting transcription factors: key players associated with abiotic stress responses in plants. Plant Mol Biol Rep 32:303-317

27. Liu, JY, Shi, YT, Yang SH (2018) Insights into the regulation of C-repeat binding factors in plant cold signaling. J Integr Plant Biol 60:80-795

28. López-Barón N, Gu Y, Vasanthan T, Hoover R (2017) Plant proteins mitigate in vitro wheat starch digestibility. Food Hydrocolloid 69:19-27

29. Mallory AC, Dugas DV, Bartel DP, Bartel B (2004) MicroRNA regulation of NAC-Domain targets is required for proper formation and separation of adjacent embryonic, vegetative, and floral organs. Curr Biol 14:1035-1046

30. Masahiro K, Kazuko Y, Hideo T, Shinozaki K (1993) Structure and expression of two genes that encode distinct drought-inducible cysteine proteinases in Arabidopsis thaliana. Gene 129:175-182

31. Mercedes DM, Blanca VA, Estrella SM, Pablo GM, Mar-tinez M and Diaz I (2016) Plant senescence and proteolysis: two processes with one destiny. Genet Mol Biol 39:329-338

32. Milla MAR, Townsend J, Chang IF, Cushman JC (2006) The ArabidopsisAtDi19 gene family encodes a novel type of Cys2/His2 zinc-finger protein implicated in ABA-independent dehydration, highsalinity stress and light signaling pathways. Plant Mol Biol 61:13-30

33. Mulholland BJ, Taylor IB, Jackson AC, Thompson AJ (2003) Can ABA mediate responses of salinity stressed tomato. Environ Exp Bot 50:17-28

34. Munné-Bosch S, Alegre L (2004) Die and let live: leaf senescence contributes to plant survival under drought stress. Funct Plant Biol 31:203-216

35. Paireder M, Mehofer U, Tholen S, Porodko A, Schahs P, Maresch D, Biniossek ML, van der Hoorn RAL, Lenarcic B, Novinec M, Schilling O, Mach L (2016) The death enzyme CP14 is a unique papain-like cysteine proteinase with a pronounced S2 subsite selectivity. Arch Biochem Biophys 603:110-117

36. Park S, Lee CM, Doherty CJ, Gilmour SJ, Kim Y, Thomashow MF (2015) Regulation of the Arabidopsis $C B F$ regulon by a complex low-temperature regulatory network. Plant J 82:193-207

37. Puranik S, Sahu PP, Srivastava PS, Prasad M (2012) NAC proteins: regulation and role in stress tolerance. Trends Plant Sci 17:369-381

38. Qin LX, Li Y, Li DD, Xu WL, Zheng Y, Li XB (2014) Arabidopsis drought-induced protein Di19-3 participates in plant response to drought and high salinity stresses. Plant Mol Biol 86:609-625

39. Rawlings ND, Barrett AJ (2010) MEROPS: the peptidase database. Nucleic Acids Res 28:323-325

40. Shi Y, Tian S, Hou L, Huang X, Zhang X, Guo H, Yang S (2012) Ethylene signaling negatively regulates freezing tolerance by repressing expression of CBF and Type-A ARR genes in Arabidopsis. Plant Cell 24:2578-2595

41. Stockinger EJ, Gilmour SJ, Thomashow MF (1997) Arabidopsis thaliana CBF1 encodes an AP2 domain-containing transcriptional activator that binds to the C-repeat/DRE, a cis-acting DNA regulatory element that stimulates transcription in response to low temperature and water deficit. $P$ Natl Acad Sci USA 94:1035-1040 
42. Sun TT, Cen GL, You CH, Lou WY, Wang ZT, Su WH, Wang WJ, Li DM, Que YX, Su YC (2020) ScAOC1, an allene oxide cyclase gene, confers defense response to biotic and abiotic stresses in sugarcane. Plant Cell Rep 39:1785-1801

43. Takada S, Hibara K, Ishida T, Tasaka M (2001) The cup-shaped cotyledon1 gene of Arabidopsis regulates shoot apical meristem formation. Development 128:1127-1135

44. Tang MF, Xu C, Cao HH, Shi Y, Chen J, Chai Y, Li ZG (2021) Tomato calmodulin-like protein SICML37 is a calcium $\left(\mathrm{Ca}^{+}\right)$sensor that interacts with proteasome maturation factor SIUMP1 and plays a role in tomato fruit chilling stress tolerance. J Plant Physiol 258:153373

45. Thomashow MF (2010) Molecular basis of plant cold acclimation: insights gained from studying the CBF cold response pathway. Plant Physiol 154:571-577

46. Usui M, Tanaka S, Miyasaka H, Suzuki Y, Shioi Y (2007) Characterization of cysteine protease induced by oxidative stress in cells of Chlamydomonas sp. strain W80. Physiol Plantarum 131:519526

47. Wang W, Vignani R, Scali M, Cresti M (2006) A universal and rapid protocol for protein extraction from recalcitrant plant tissues for proteomic analysis. Electrophoresis 27:2782-2786

48. Wang Y, Xin HP, Fan PG, Zhang JS, Liu YB, Dong Y, Wang ZM, Yang YZ., Zhang Q, Ming R, Zhong GY, Li SH, Liang ZC (2021) The genome of Shanputao (Vitis amurensis) provides a new insight into cold tolerance of grapevine. Plant J 105:1495-1506

49. Xu ZF, Chye ML, Li HY, Xu YL, Yao K (2003) G-box binding coincides with increased Solanum melongena cysteine proteinase expression in senescent fruits and circadian-regulated leaves. Plant Mol Biol 51:9-19

50. Yang JC, Zhang JH, Wang ZQ, Zhu QS, Liu LJ (2003) Involvement of abscisic acid and cytokinins in the senescence and remobilization of carbon reserves in wheat subjected to water stress during grain filling. Plant Cell Environ 26:1621-1631

51. Yang T, Poovaiah BW (2003) Calcium/calmodulin- mediated signal network in plants. Trends Plant Sci 8:505-512

52. Ye YY, Ding YF, Jiang Q, Wang FJ, Sun JW, Zhu C (2017) The role of receptor-like protein kinases (RLKs) in abiotic stress response in plants. Plant Cell Rep 36:1-8

53. Yin FL, Zeng YL, Ji JY, Wang PJ, Zhang YF, Li WH (2021) The Halophyte Halostachys caspica AP2/ERF Transcription Factor HcTOE3 Positively Regulates Freezing Tolerance in Arabidopsis. Front Plant Sci 12:638788

54. Yu Y, Xu W, Wang J, Wang L, Yao W, Xu Y, Ding J, Wang Y (2013) A core functional region of the RFP1 promoter from Chinese wild grapevine is activated by powdery mildew pathogen and heat stress. Planta 237:293-303

55. Zang QW, WangCX, Li XY, Guo ZA, Jing RL (2010) Isolation and characterization of a gene encoding a polyethylene glycol-induced cysteine protease in common wheat. J Biosciences 35:379-388 
56. Zhang BH, Gao X, Yang MM, Wang XG, He QM, Yao XL, Li XY, Zhao WC,Dong J (2019) Cloning of TaCP3 gene and its expression in abiotic stress. Journal of Triticeae Crops 39:513-519 (in Chinese)

57. Zheng L, Chen S, Xie L, Zhu CL, Liu MY, Han XJ, Qiao GR, Jiang J, Zhuo RY, Qiu WM, He ZQ (2018) Overexpression of cysteine protease gene from, Salix matsudana, enhances salt tolerance in transgenic Arabidopsis. Environ Exp Bot 147:53-62

\section{Figures}

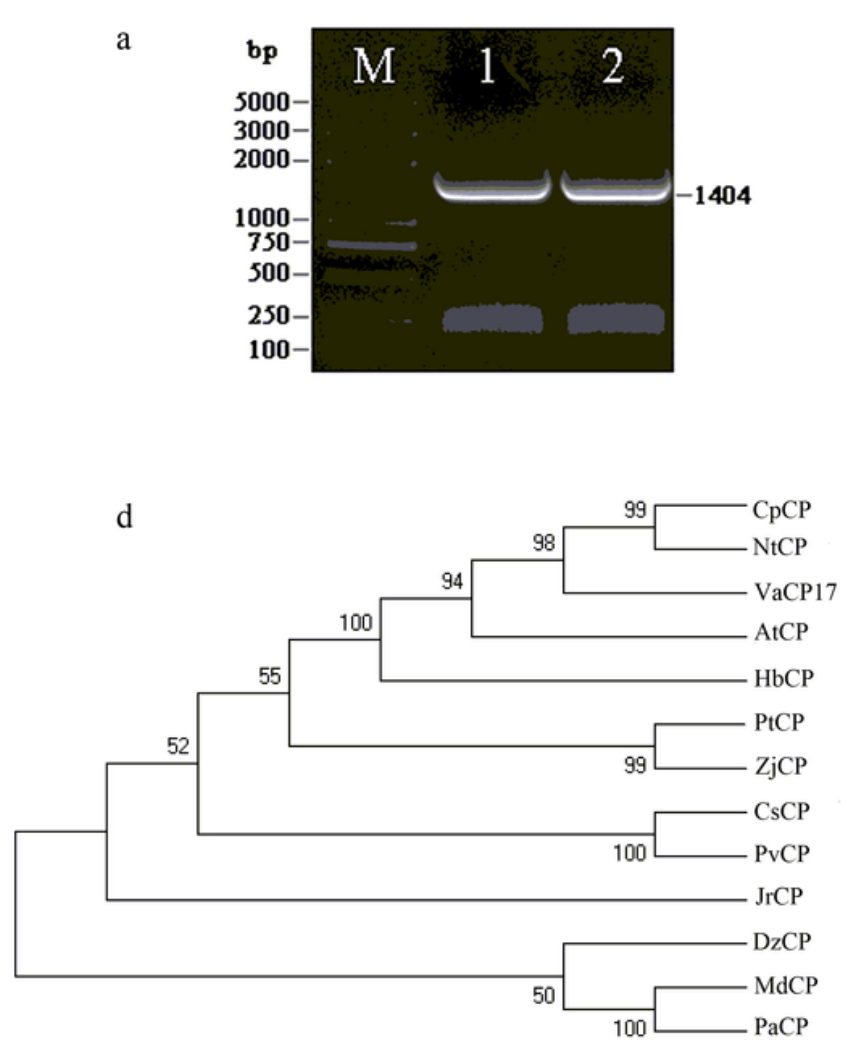

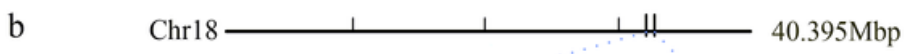

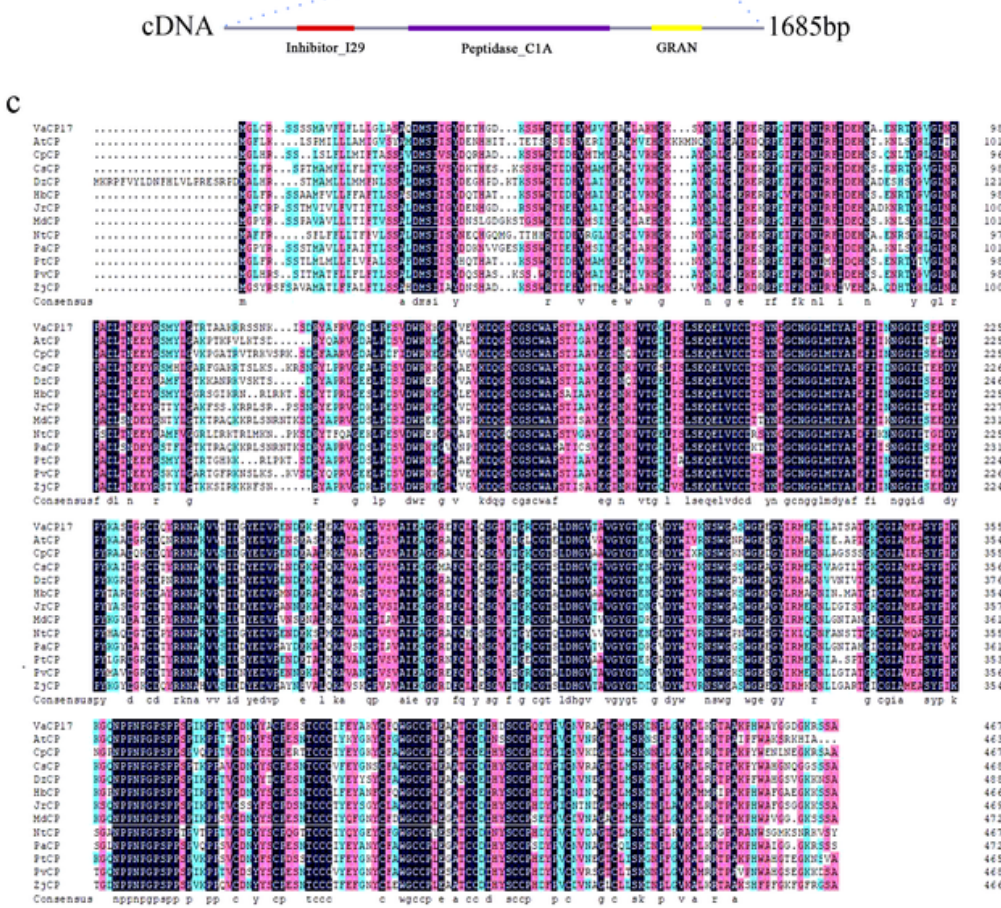

Figure 1

Cloning and sequence analysis of VaCP17 isolated from Vitis amurensis accession 'Shuangyou' (a) Cloning of VaCP17 from V. amurensis acc. 'Shuangyou'. M. DNA marker III; 1-2. Independent VaCP17 amplification products. (b) Chromosomal location of VaCP17 and conserved domains within the protein. (c) Multiple sequence alignmnet of VaCP17 with strongly homologous CPs from other plant species. (d) Phylogenetic analysis of VaCP17 and other plant CPs. The accession numbers are as follows: AtCP, Arabidopsis thaliana NP_568620.1; CpCP, Carica papaya XP_021903335.1; CsCP, Citrus sinensis XP_006473275.1; DzCP, Durio zibethinus XP_022714907.1; HbCP, Hevea brasiliensis XP_021650530.1; JrCP; Juglans regia XP_018852257.1; MdCP, Malus domestica XP_008365104.2; NtCP, Nicotiana tabacum XP_016451793.1; PaCP, Prunus avium, XP_021820221.1; PtCP, Populus trichocarpa XP_006374886.1; PvCP, Pistacia vera XP_031259300.1; ZjCP, Ziziphus jujuba XP_015884332.1. 


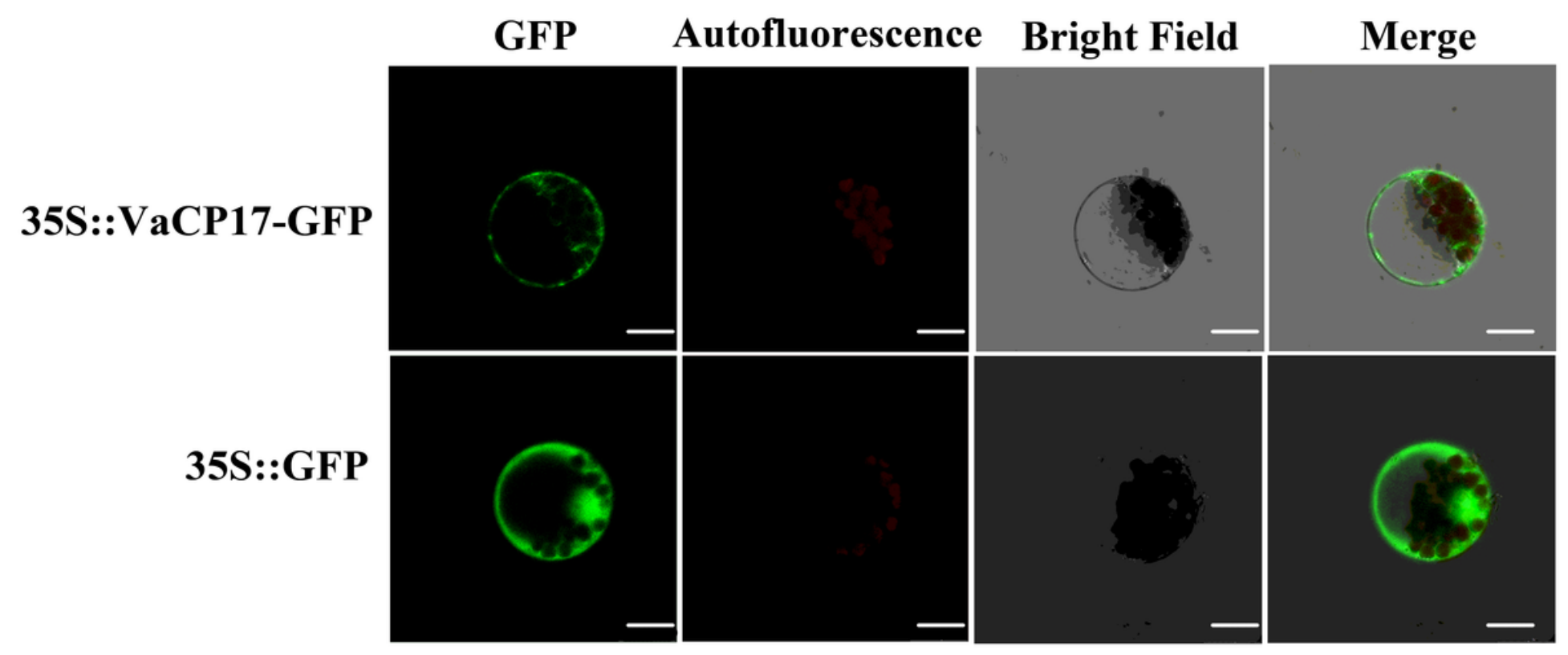

Figure 2

Subcellular localization of VaCP17 35S::VaCP17-GFP was transformed into Arabidopsis protoplasts by a PEG mediated method. Cells were observed using fluorescent microscopy. Bars, $20 \mu \mathrm{m}$. 


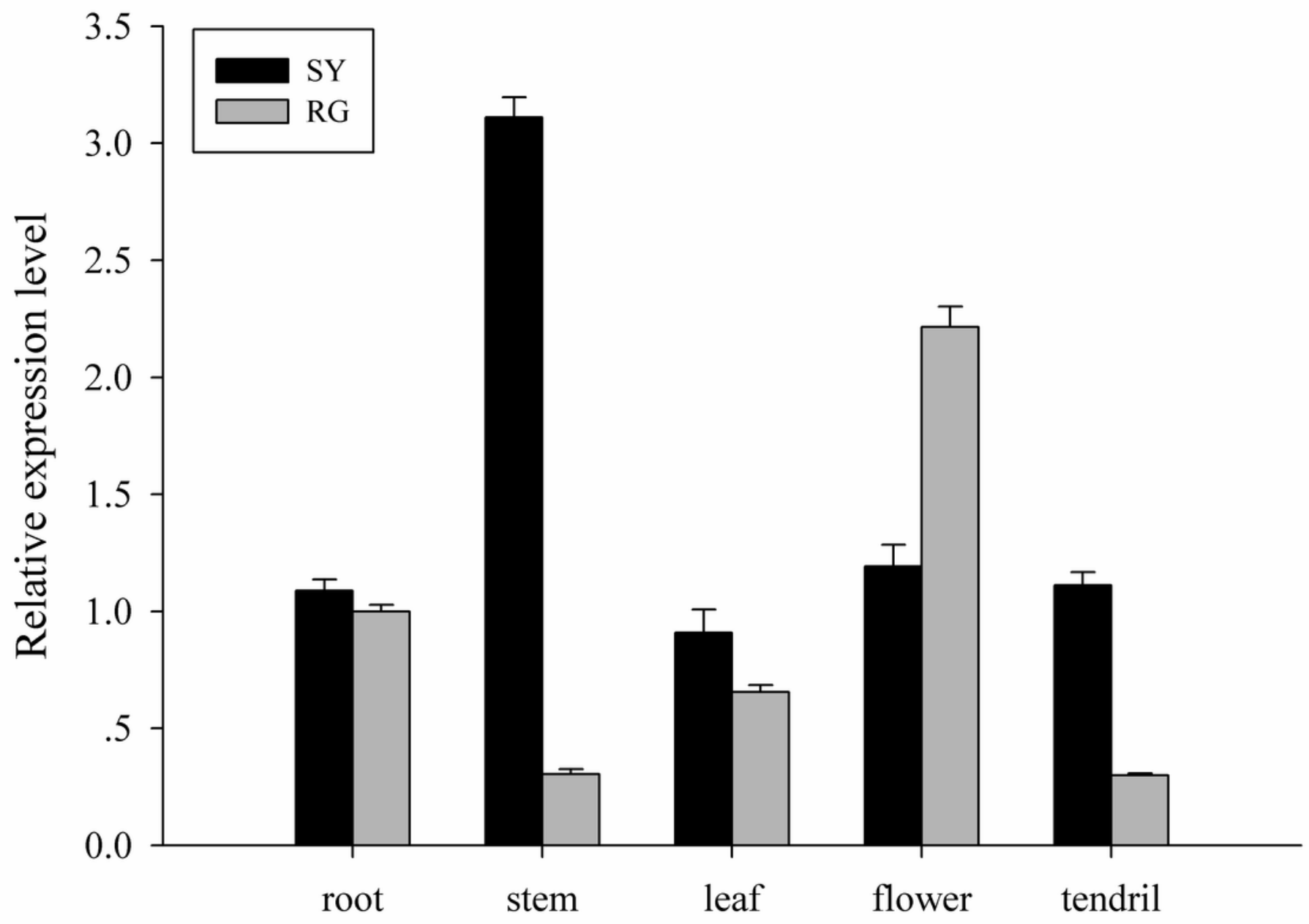

Figure 3

Developmental expression patterns of CP17 in 'Shuangyou' and 'Red Globe' SY. 'Shuangyou'; RG. 'Red Globe'.

a

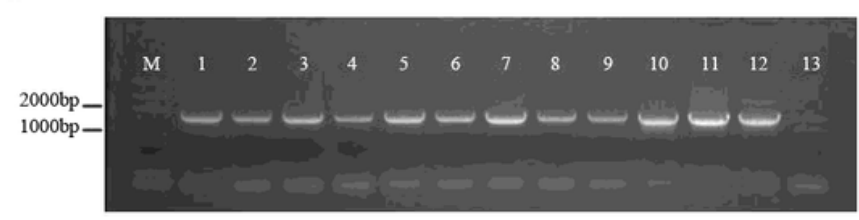

c

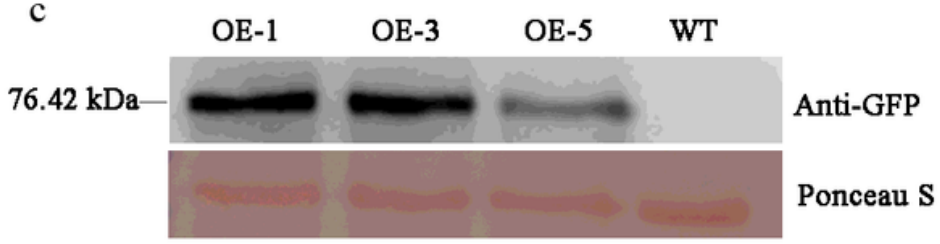

b

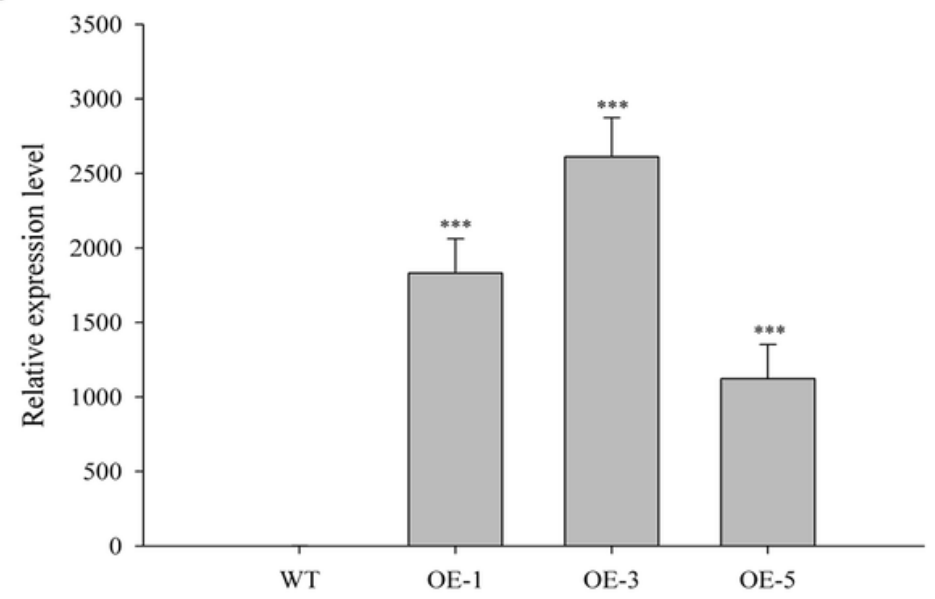




\section{Figure 4}

Identification and analysis of 35S::VaCP17-GFP Arabidopsis (a) qRT-PCR analysis of VaCP17 expression

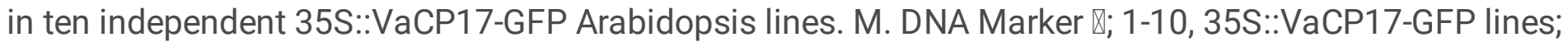
11-12, positive plasmid control; 13. Negative control. (b) Quantification of qRT-PCR analysis for lines OE1, OE-3, and OE-5. Non-transgenic Arabidopsis was used as a negative control. (c) Western blot analysis of VaCP17-GFP protein expression in lines OE-1, OE-3, and OE-5. WT, non-transgenic control. The predicted molecular mass for VaCP17 is $51.42 \mathrm{kDa}$, while that for GFP protein is $25 \mathrm{kDa}$, so the total expected molecular mass of VaCP17-GFP protein is $76.42 \mathrm{kDa}$.

a
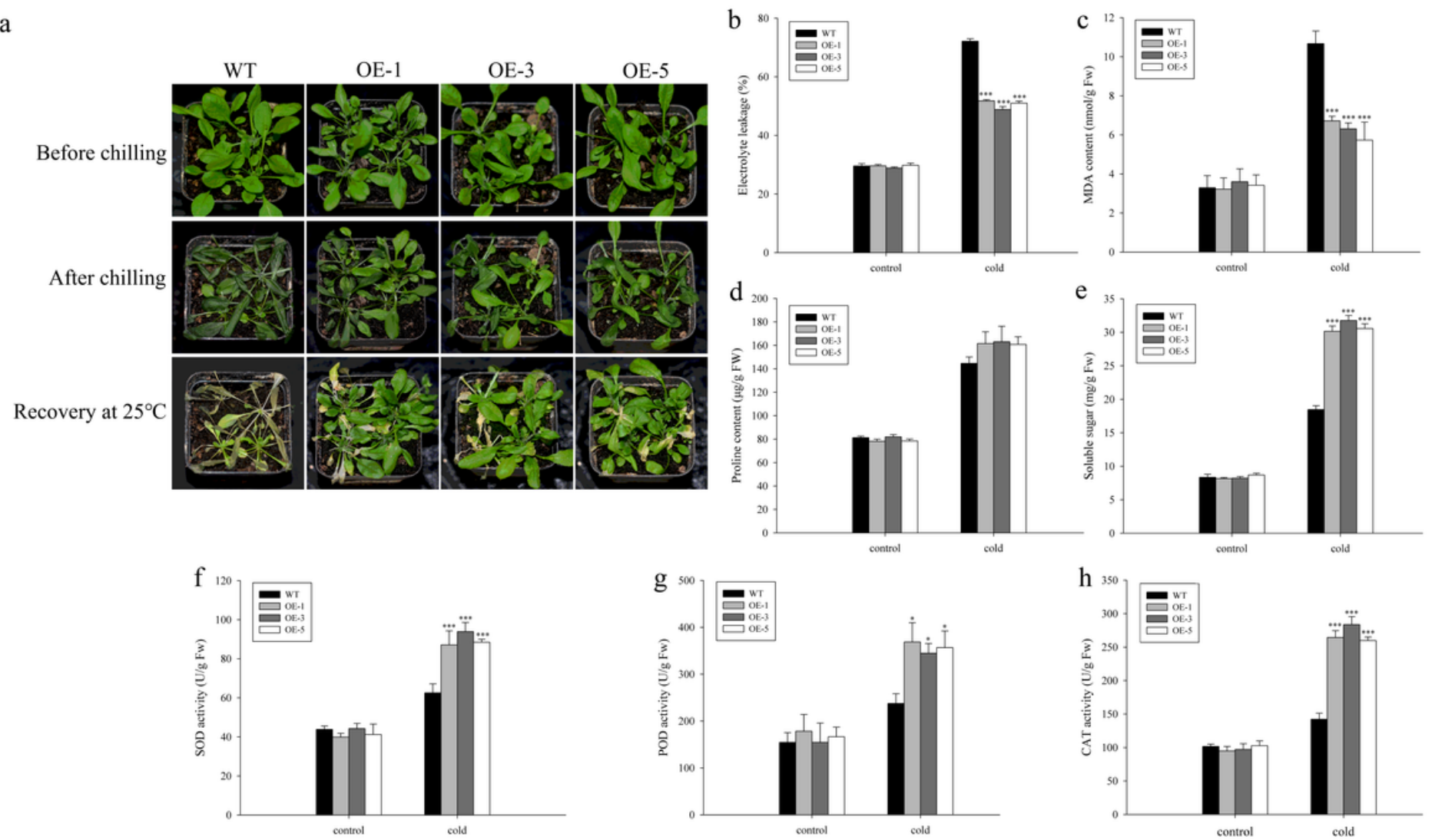

\section{Figure 5}

Heterologous expression of VaCP17 in Arabidopsis enhances cold resistance. (a) Plants of nontransgenic control (WT) and 35S::VaCP17-GFP lines (OE-1, OE-3 and OE-5) before and after a chilling treatment, and after recovery at $25^{\circ} \mathrm{C}$. (b) Electrolyte leakage and (c) malondialdehyde (MDA) content. (d) Proline content and (e) soluble sugar content. (f-h) Activities of SOD, POD and CAT, respectively. Data are the mean $( \pm S D)$ of three biological replicates. Significance was analyzed with SPSS using the LSD and Duncan methods of one-way ANOVA $\left({ }^{*} \mathrm{P}<0.05 ;{ }^{* *} \mathrm{P}<0.01, * \star \star \mathrm{P}<0.001\right)$ 


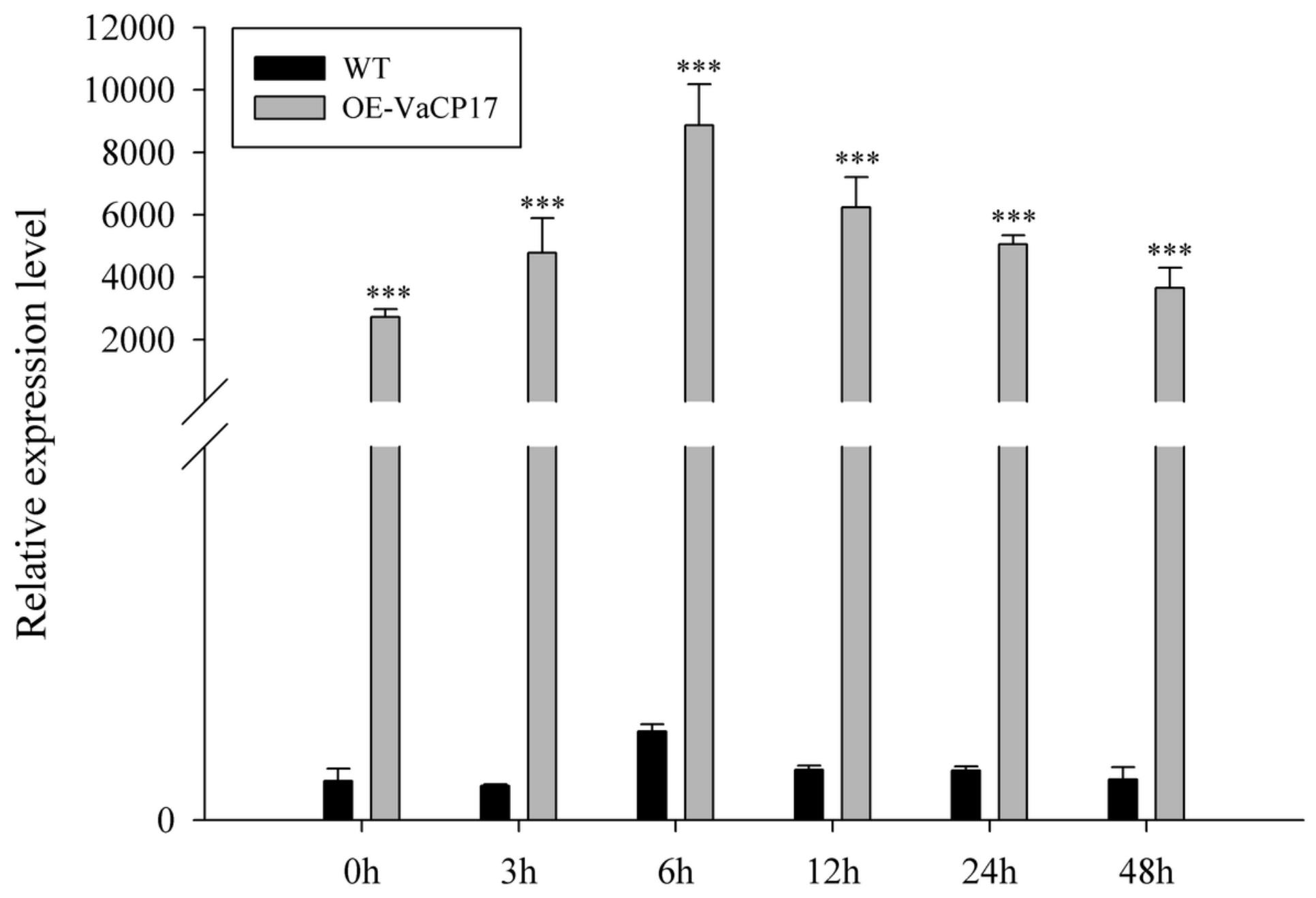

Figure 6

The relative expression level of VaCP17 in 35S::VaCP17-GFP and control Arabidopsis under cold stress OE-VaCP17. transgenic Arabidopsis thaliana line OE-3; WT, non-transgenic control Arabidopsis. Data are the mean $( \pm S D)$ of three biological replicates. Significance was analyzed with SPSS using the LSD and Duncan methods of one-way ANOVA ( $\left.{ }^{*} P<0.05 ;{ }^{* *} P<0.01,{ }^{* * *} P<0.001\right)$. 

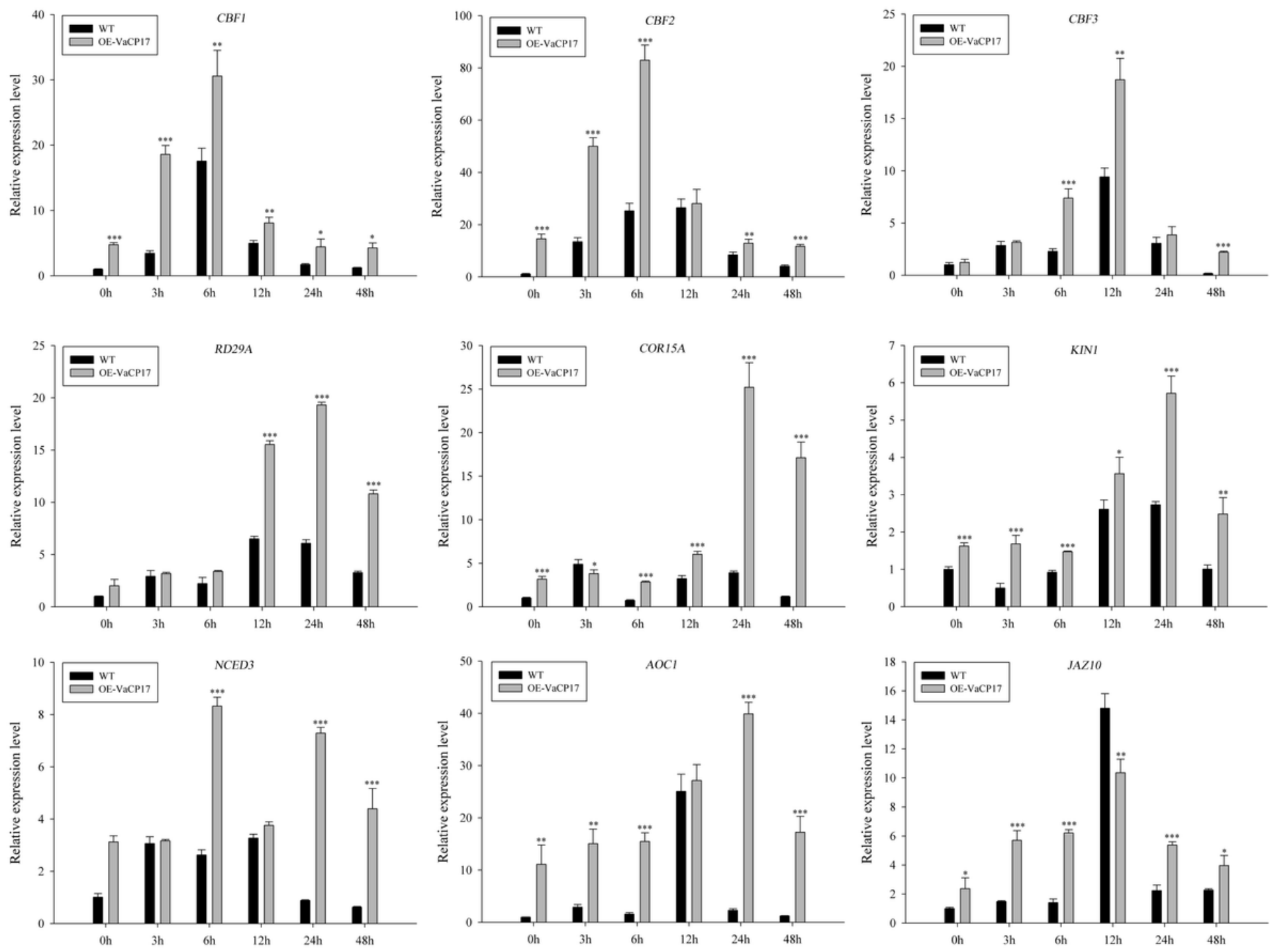

Figure 7

Relative expression levels of cold resistance-related genes in 35S::VaCP17-GFP and control Arabidopsis under cold stress OE-VaCP17. transgenic Arabidopsis thaliana line OE-3; WT. non-transgenic control Arabidopsis. Data are the mean $( \pm S D)$ of three biological replicates. Significance was analyzed with SPSS using the LSD and Duncan methods of one-way ANOVA ( $\left.{ }^{\star} P<0.05 ;{ }^{* \star P}<<0.01,{ }^{\star \star *} \mathrm{P}<0.001\right)$. 
a
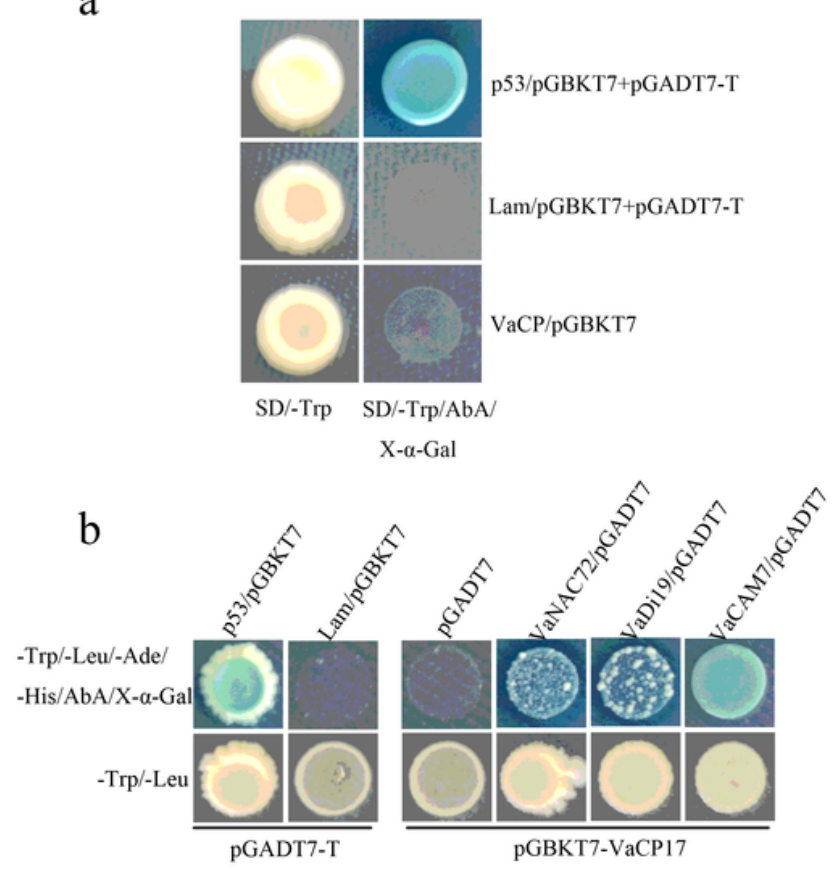

c

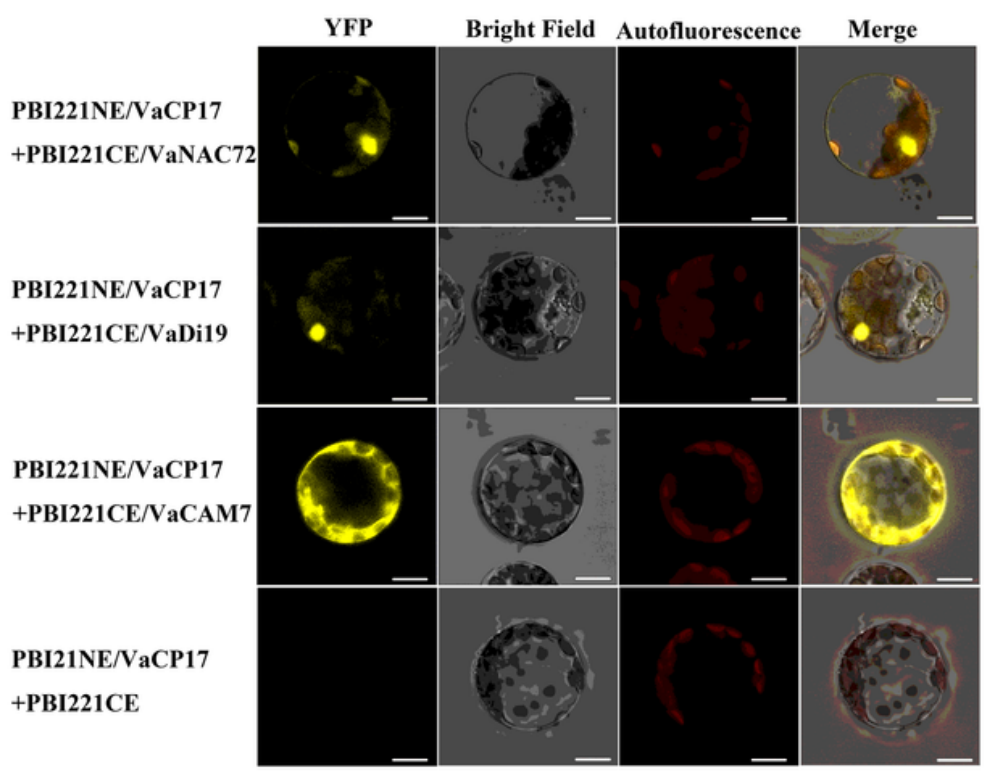

\section{Figure 8}

Confirmation of VaCP17-interacting proteins (a) Transcriptional activation analysis of VaCP17 in yeast. p53/pGBKT7 and Lam/pGBKT7 were co-transformed with pGADT7-T as positive and negative controls, respectively. The recombinant vector VaCP17/pGBKT7, positive control and negative control were placed on SD/-Trp and SD/-Trp/AbA/X-a-gal solid medium and cultured at $28^{\circ} \mathrm{C}$ for $3 \mathrm{~d}$. (b) $\mathrm{Y} 2 \mathrm{H}$ assay of the interaction between VaCP17 and VaNAC72, VaDi19, or VaCAM7. VaCP17/pGBKT7 and X/pGADT7 were cultured on solid medium SD/-Trp/-Leu and SD/-Trp/-Leu/-Ade/-His/AbA/X-a-gal for 2 4 d. The empty pGADT7 vector co-transformed with VaCP17/pGBKT7 was used as a control. p53/pGBKT7 and Lam/pGBKT7 were co-transformed with pGADT7-T as positive and negative controls, respectively. (c) BiFC assay to test the interaction between VaCP17 and VaNAC72, VaDi19, or VaCAM7 in vivo. Plasmids were co-transformed into Arabidopsis protoplasts and cultured at $22^{\circ} \mathrm{C}$ under weak light for about $18 \mathrm{~h}$, and YFP signal was observed under a confocal microscope. PBI221NE/VaCP17 was co-transformed with $\mathrm{PBI} 21 \mathrm{CE} / \mathrm{X}$ as test group. Empty PBI221CE was co-transformed with PBI221NE/VaCP17 as a control. Bars, $20 \mu \mathrm{m}$.

\section{Supplementary Files}

This is a list of supplementary files associated with this preprint. Click to download.

- Supplementarytable1.docx 